\title{
Research and Application of Micro-Services Framework for Public Information of Waterway
}

\section{Yu Li}

No. 64, Public Road, Gulou District, Nanjing, Jiangsu, China, Yangtze River Nanjing Waterway Bureau, 210011, China

Keywords: Micro-service, Public Information of waterway, REST API.

\begin{abstract}
Public information of waterway was usually serviced by waterway management departments in conventional means such as official websites, special Apps and so on. These service ways lead to that it is hard to make full using of waterway data resources in users' own application scenarios and systems. This paper designs a four-tier micro-service framework for waterway public information services, it including data layer, interface layer, application layer and business application client layer. Public information of waterway collected from the digital waterway systems was classified into different type data resources, which were then serviced by REST-based APIs dividedly complying with the rules of micro-service. And, now the waterway public information services had be utilized by several third-part application systems in multiple scenarios. The results showed that the micro-service framework is an effective way to publish the waterway public information, and improve the utilization efficiency of data resources from digital waterway systems.
\end{abstract}

\section{Introduction}

With the popularity of the Internet, the waterway management department generally uses various information platforms to publish channel public information, such as official websites, APPs, and social media. This kind of information service method is presented in a specific application scenario through a specific platform, and it is difficult to fully utilize the role of public information resources.

Sun Tengda [1] and other researches have concluded that the inland river channel information in the Yangtze River Delta region is highly concerned, and users are more inclined to intuitive passive services. Due to the scattered data resources, a data sharing mechanism needs to be established. In the research of the key technical system of the Yangtze River intelligent navigation channel, Liu Huaihan [2] proposed a variety of channel information service methods including interfaces to promote the sharing of channel information services. Li Xuexiang [3] proposed a service method using the "Internet +" thinking.

Different from the above methods, this paper designed and practiced a micro-service framework for waterway public information services.

\section{Framework Design}

\section{Architecture}

The framework is composed of three parts: the waterway business platform, the service platform and the third-party application platform (Figure 1):

1) Waterway business platform: The waterway management department conducts business management in the waterway business platform (with the digital waterway systems). In order to ensure the safety of the production database, internal and external network isolation is often implemented. The production database is used as a service data source, and is synchronized to the database server in the external network through the firewall and the gatekeeper [4].

2) Service platform: The mainstream often adopts cloud service solutions. The service platform obtains data from the pre-database and rebuilds the database cluster to form an external service data center, and deploys related data and application services in a micro-service architecture to open to the entire social application platform. 
3) Third-party application platform: After obtaining the corresponding public information of the channel, the authorized application platform can combine the application scenarios to realize the channel information service in each characteristic application front end.

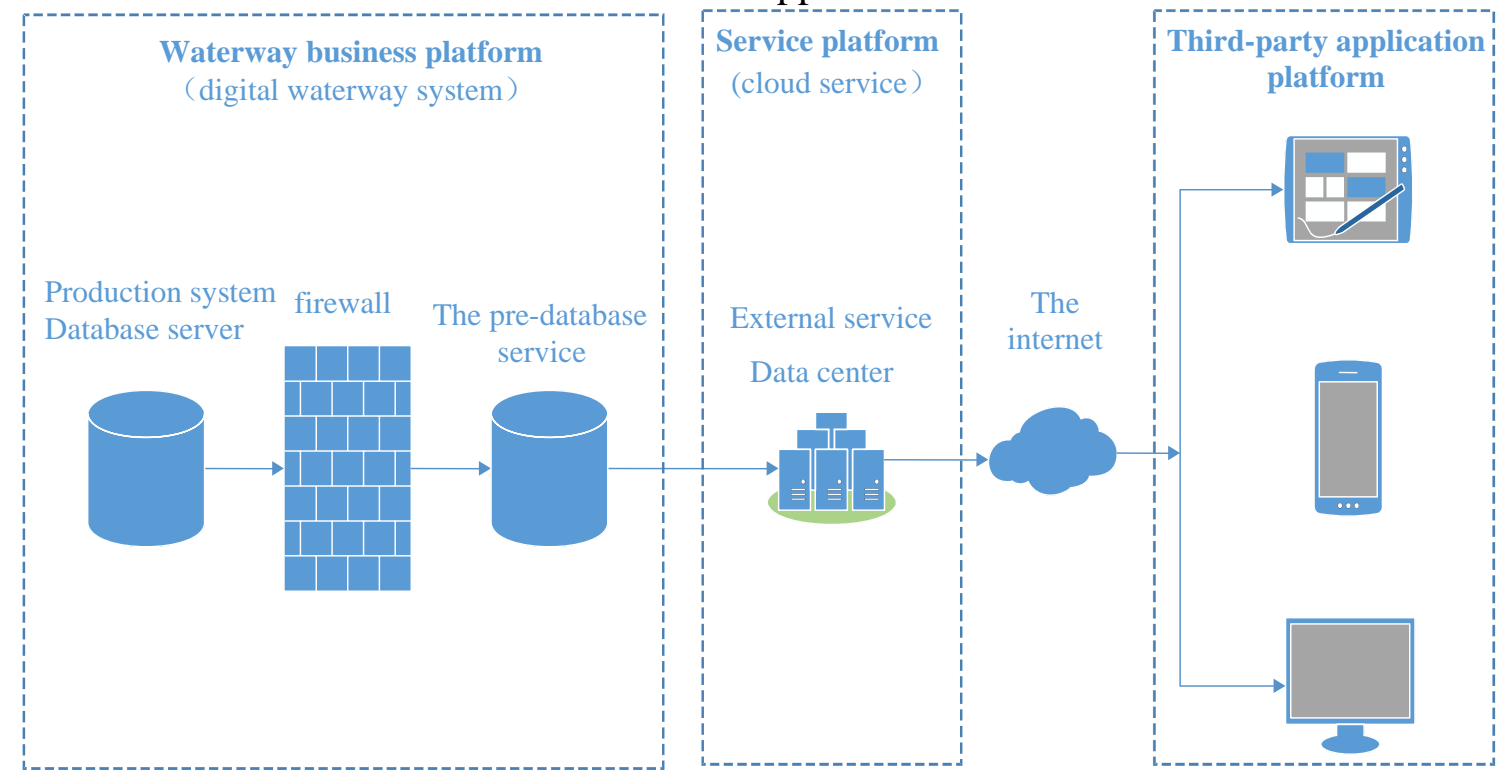

Figure 1. Overall architecture

\section{Logical Structure}

The logical structure is divided into data layer, micro service interface layer, application layer and client layer:

1) Data layer: synchronize from production data to external database in data synchronization mode. Subdivide the channel public information data and fully decouple it. The subdivided databases are deployed in the service data center. A MySQL database was used to synchronize data from the external database through a batch program developed by Spring Batch and Quartz [5].

2) Micro-service interface layer: subdivided into user data, water level, navigation mark, channel scale, channel notice and electronic channel map micro-service. Each data micro-service defines an interface protocol based on REST style and uses an HTTP-based JSON format open interface for easy access and utilization by all third parties.

3) Application layer: interface application and management platform. The interface application platform provides an interface application interface for the third party, and the interface management platform provides the channel management department with an interface for accepting, approving, and managing the interface application.

4) Client layer: The third party signs an agreement with the waterway management department to obtain the KEY for data utilization license. When requesting data through the interface, the KEY is provided for interface verification, and after obtaining the data, the public information of the channel is comprehensively utilized according to the user's needs.

\section{Micro-service API}

\section{Spring-based REST Web Services}

As shown in Figure 2, spring Boot based solution was used to implement the service interface in the following structure: spring boot + mybatis + redis + druid + mysql. And the WMS and WFS service of the electronic channel map is especially implemented based on GeoServer. 


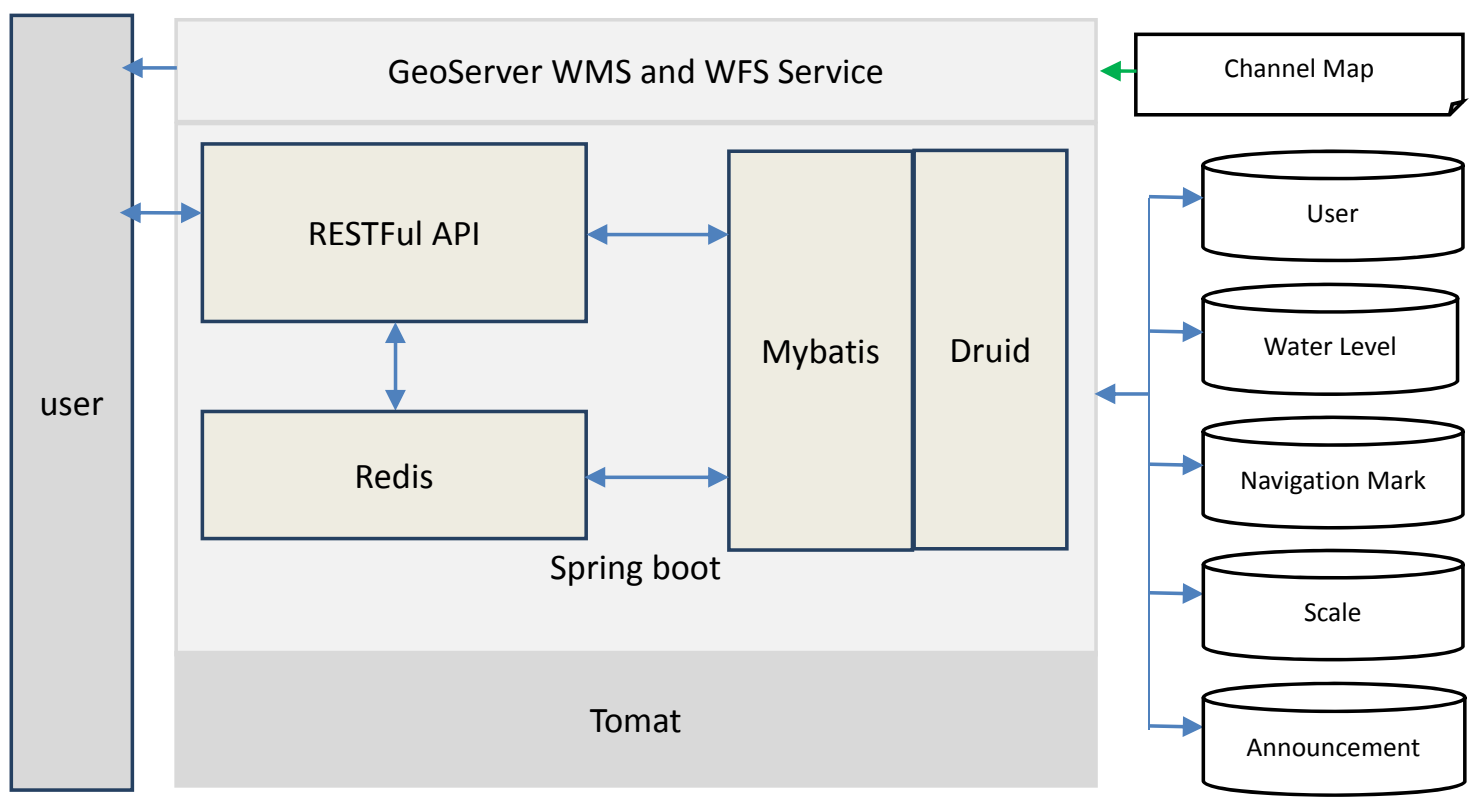

Figure 2. Spring-based REST Web service implementation framework

Among them, the data center uses mysql as the database, and according to different service categories, the data is divided into different libraries, and the multi-data source is integrated by Mybatis+Druid. Integrate MyBatis as an ORM (Object Relational Mapping) framework using Spring boot. Because the expected concurrent request volume is relatively large, and the relational database query efficiency is poor, Redis is used as the secondary cache of Mybatis to cache business data and improve interface performance. All applications in this study were deployed using Tomcat clustering and were stateless.

\section{Interface Security}

Authentication and authorization are performed by means of user access_token + sign. That is, the corresponding access_token (key+secret) is assigned to the third-party application platform. When each application invokes the service API, it needs to perform signature verification on the request parameters. The verification uses the md5 parameter signature method: according to the request parameter name, all the request parameters are sorted according to the parameter name ASCII code from small to large, such as: aong=1\&brg=2. Add the secret to the end of the parameter string and perform MD5 encryption. The encrypted string needs to be capitalized to get the signature Sign.

\section{Interface Data Structure}

This article has developed various micro-service interface specifications based on the project. The interface has three necessary parameters: organizationId, timeStamp and sign, which are used to authenticate and authorize the caller. Interfaces can have their own parameters, and the return values of all interfaces have a uniform JSON format:

Result: indicates whether the interface is successfully called;

Data: The data set for the query exists in JSON form. Different interfaces will have a collection of data in different formats;

Time: indicates when the server returns data;

errorCode: indicates the server error code;

errorMsg: indicates specific error information;

Total: indicates the number of returned data, that is, the number of data in data.

\section{Third-party Application}

The waterway public information micro-service APIs has been utilized by several third-party platforms from different application scenarios including integrated queries, ship navigation, ecommerce, and social platforms [6]. 
The Figure3 showed a case of a famous shipping services website, www.shipxy.com. As the figured showed, the website overlayed the Yangtze River channel map attained from our WMS microservice API onto the Google map, and the merged map provided more friendly feeling for shipping users. WFS micro-service API was also applied that can provide the query functions for channel map features. In addition, the website also accesses other type micro-service APIs such those for dynamic information of channel navigational mark, water level and channel maintaining scale, they were all integrated into the map platform, and provided shipping users the realtime channel information of Yangtze River. Besides websiete, the company of www.shipxy.com can also applied the waterway public information into their APPs or other productions.

By the thirt-party applications, the waterway public information can be accessed by more users in more methods. So, it is meaningful for promoting the utilization effiecency of the archivements of digital waterway systems.

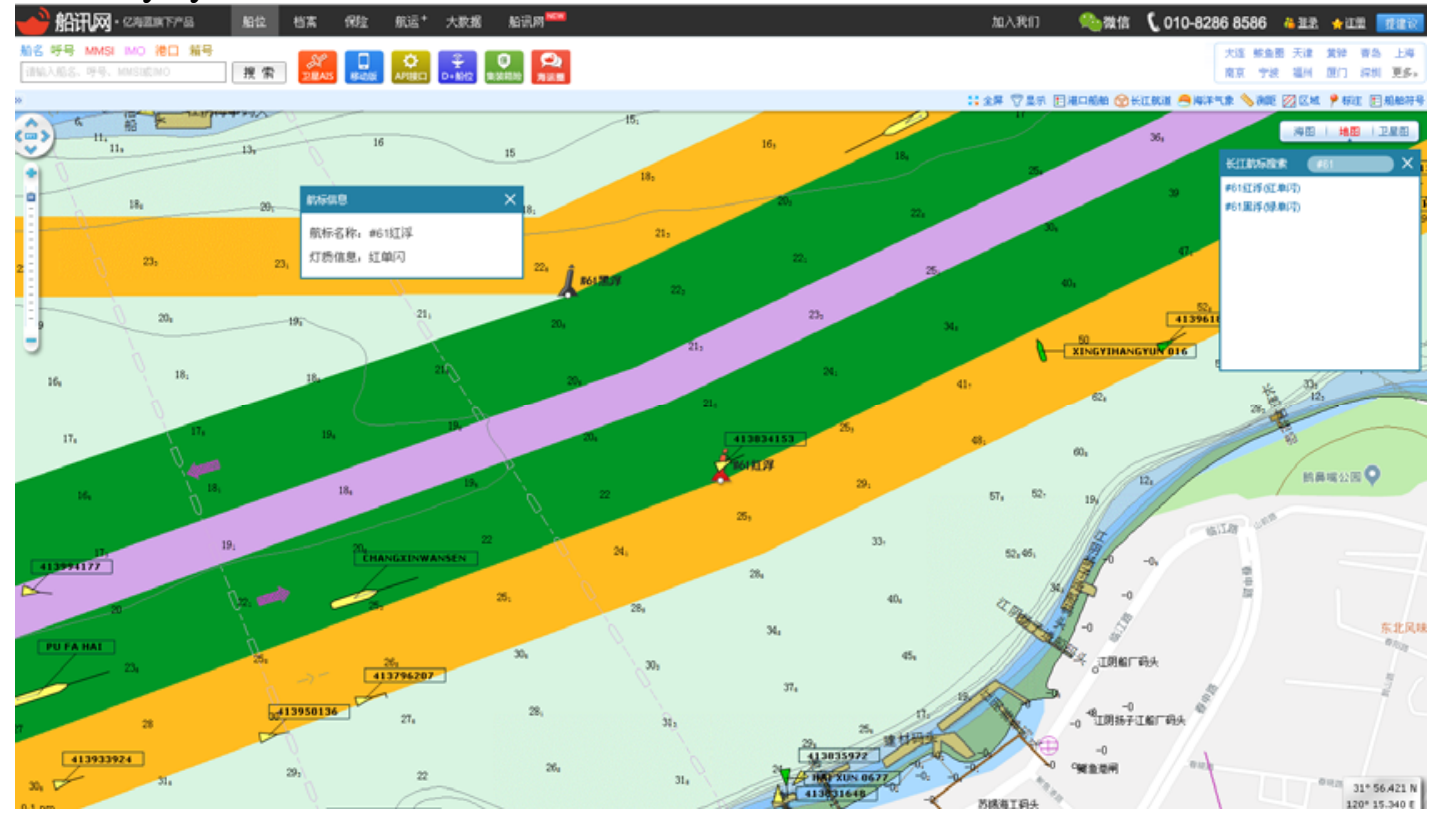

Figure 3. A third-party application (www.shipxy.com)

\section{Summary}

This paper studies a micro-service-based waterway public information service framework, which constitutes an open service system composed of waterway business platform, service platform and third-party application platform. The service structure of the system is divided into a data layer, a micro service interface layer, an application layer, and a client layer. The micro service interface was issued as standardized REST web service, which can be utilized easily by the third-partys to their own application scenarios and systems.

Based on the research, the Yangtze River downstream channel micro-service system was successfully implemented, and it had cooperated with serveral thirt-party Internet platforms for application, and achieved remarkable social benefits.

\section{Acknowledgement}

*Project Fund: Science and Technology Project of Yangtze River Nanjing Waterway Bureau "Nanjing the Lower Yangtze River Channel Public Information Microservices and Application Demonstration Research” (2017002), “Public Information Microservices of Reaches of the Lower Yangtze River (Phase II)” (2018002). 


\section{References}

[1] Sun Tengda, Liu Zhehui, Qiu Jianhua. Demand Analysis for River Information Services in Yangtze River Delta Region[J]. Navigation of China.2014,37(03):5-9+20.

[2] LIU Huaihan, LI Xuexiang. YANG Pinfu, GUO Tao.System of key technology of Yangtze River intelligent waterway[J]. Port \& Waterway Engineering,2014(12):6-9.

[3] Li Xuexiang. Thinking on the Development of Changjiang Digital Waterway in the "Internet + " Age[J]. Geomatics \& Spatial Information Technology,2016,39(10):1-4+10.

[4] Zhou Junan, Liu Jialun, Liu Mingjun. Research on the Information Publish Mode and Publish Mode in Yangtze River Main Channel[J]. Journal of Wuhan University of Technology (Transportation Science \& Engineering), 2012,36(05):1017-1020.

[5] Jin Shisheng, Li Jue. Design and implementation of data migration processing system based on Spring Batch [J]. China New Telecommunications, 2016,18(20):54-55.

[6] Mu Qiang. Research on Yangtze River Channel Microservice Based on WeChat Small Program [J]. China Water Transportation (Science \& Technology for Waterway),2018(04):66-70. 